\title{
High fat meals increases postprandial fat oxidation rate but not postprandial lipemia
}

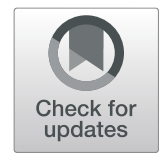

Chih-Hui Chiu ${ }^{*}$, Tsung-Jen Yang ${ }^{2}$, Che-Hsiu Chen ${ }^{3}$ and Ming-Jing Zeng ${ }^{1}$

\begin{abstract}
Background: This study investigated the effects of ingesting meals with the same calorie intake but distinct nutritional contents after exercise on postprandial lipemia the next day.

Methods: Eight healthy male participants completed two 2-day trials in a random order. On day 1, the participants underwent five 12 min bouts of cycling exercise with a bout of higher intensity exercise $(4 \mathrm{~min})$ after each and then a bout of lower intensity cycling $(2 \mathrm{~min})$. The total exercise time was $90 \mathrm{~min}$. After the exercise, the participants ingested three high-fat or low-fat meals. On Day 2, the participants were asked to rest in the laboratory and ingest a high-fat meal. Their postprandial reaction after a high-fat meal was observed.

Results: Postprandial triglyceride concentrations in the high-fat diet trial and low-fat diet trial exhibited nonsignificant differences. Total TG AUC were no significantly different on HF trial and LF trial (HF: $6.63 \pm 3.2$; LF: $7.20 \pm 3.4 \mathrm{mmol} / \mathrm{L}^{*} 4 \mathrm{~h} . p=0.586$ ). However, the postprandial fat oxidation rate total AUC (HF: $0.58 \pm 0.1$; LF: $0.39 \pm 0.2 \mathrm{~g} / \mathrm{min}^{*} 4 \mathrm{~h} . p=0.045$ ), plasma glucose, and insulin concentration of the high-fat trial were significantly higher than those of the low-fat trial.

Conclusions: This study revealed that meals with distinct nutritional contents after a 90-min exercise increased the postprandial fat oxidation rate but did not influence the postprandial lipemia after a high-fat meal the next day.
\end{abstract}

Keywords: Intramuscular triglycerides, Insulin sensitivity, Exercise

\section{Introduction}

Elevated postprandial triglyceride (TG) concentrations have been suggested to significantly increase the risk of metabolic disease [1]. A single session of exercise can decrease postprandial TG concentrations the next day $[2,3]$. Numerous studies have found that energy expenditure during exercise may play a vital role in postprandial TG response [4, 5]. Exercise decreases postprandial lipemia the next day by enhancing the lipoprotein lipase (LPL) activity [6], increasing the postprandial fat oxidation rate [7], and improving the insulin sensitivity after exercise [8]. However, the exact mechanism underlying this phenomenon remains unknown.

Diverse types of meals with a varying nutritional content may influence postprandial TG concentration. Under nonexercise conditions, high-carbohydrate diets have been

\footnotetext{
* Correspondence: loveshalom@hotmail.com

${ }^{1}$ Department of Exercise Health Science, National Taiwan University of Sport,

No.16, Sec. 1, Shuang-Shih Rd, Taichung 404, Taiwan

Full list of author information is available at the end of the article
}

suggested to decrease the hepatic fatty acid oxidation and increase plasma TG concentration [9]. After exercise, high-carbohydrate diets induce a higher postprandial TG concentration compared with low-carbohydrate diets [10]. This may be because high-carbohydrate diets decrease postprandial fat oxidation [10]. However, high-fat (HF) postexercise meals have also been found to increase postprandial fat oxidation [11]. The relationship between a diet's varying nutritional content and postprandial fat oxidation remains unclear.

Postprandial fat oxidation may play a major role in postprandial lipemia. High-intensity interval exercise may increase postprandial fat oxidation and reduce postprandial TG concentration the next day [12, 13]. In addition, HF postexercise meals increased postprandial fat oxidation [11]. The effect of a higher postprandial fat oxidation rate induced by HF meals after exercise on postprandial TG concentration remains unclear. The objective of this study was to investigate the effects of

(C) The Author(s). 2019 Open Access This article is distributed under the terms of the Creative Commons Attribution 4.0 International License (http://creativecommons.org/licenses/by/4.0/), which permits unrestricted use, distribution, and 
ingesting HF or low-fat (LF) meals with the same calorie intake after exercise on postprandial TG concentration and postprandial fat oxidation based on an oral fat tolerance test (OFTT) the next day.

\section{Methods}

\section{Participants}

Eight healthy male participants were recruited (age $22 \pm$ $1.3 \mathrm{yr}$, height $170.1 \pm 4.7 \mathrm{~cm}$, weight $75.4 \pm 17.5 \mathrm{~kg}$; Table 1 ). No participant received professional exercise training, but had the habit of exercising two to three times a week. The participants did not present any metabolic disorders, lipemia, or other problems rendering them unfit to engage in exercise. A questionnaire was used to screen for physical activity level and any potential health issues before testing. After completely understanding the experiment, the participants signed an informed consent form. This study was approved by the Institutional Review Board of Changhua Christian Hospital (CCH IRB No 151221) in Taiwan.

\section{Design}

A crossover design approach was adopted in this study. The experiment involved two trials, namely an LF diet trial and an HF diet trial. Participants first underwent a pretest to measure their $\mathrm{VO}_{2 \max }$ and calculate the intensity of their interval training during the formal experiment. The pretest and formal experiment had to take place at least 7 days apart. The participants exercised at $66 \% \mathrm{VO}_{2 \max }$ for $90 \mathrm{~min}$ in the morning on the first day of the formal experiment. Interval training was incorporated five times during the process, and at the end of the exercise, three LF or HF meals with equal calorie intakes were administered. The experimental sequences occurred in a random order, with each test conducted at least 7 days apart from the others to avoid influences.

Table 1 The participants physiological information and fasting plasma biochemistry

\begin{tabular}{|c|c|c|c|}
\hline & LF & $\mathrm{HF}$ & $p$ value \\
\hline Body Mass Index (BMI, kg/m²) & $24.1 \pm 5.6$ & & \\
\hline Body fat (\%) & $21.0 \pm 4.7$ & & \\
\hline $\begin{array}{l}\text { Average heart rate during } \\
\text { exercise (beat/min) }\end{array}$ & $147.15 \pm 10.55$ & $148.38 \pm 8.19$ & 0.414 \\
\hline $\begin{array}{l}\text { Energy expenditure during } \\
\text { exercise (Kcal) }\end{array}$ & $987.4 \pm 142.4$ & $997.8 \pm 129.2$ & 0.527 \\
\hline $\mathrm{TG}(\mathrm{mmol} / \mathrm{L})$ & $0.93 \pm 0.48$ & $0.62 \pm 0.22$ & 0.067 \\
\hline Glucose (mmol/L) & $4.86 \pm 0.49$ & $5.01 \pm 0.44$ & 0.505 \\
\hline Insulin (pmol/L) & $1.12 \pm 0.47$ & $1.58 \pm 0.90$ & 0.077 \\
\hline NEFA (mmol/L) & $0.85 \pm 0.19$ & $0.80 \pm 0.16$ & 0.597 \\
\hline Glycerol $(\mu \mathrm{mol} / \mathrm{L})$ & $46.57 \pm 21.80$ & $47.75 \pm 14.13$ & 0.791 \\
\hline
\end{tabular}

Values are mean $\mathrm{SD}, n=8$. LF, low fat diet trial; $\mathrm{HF}$, high fat diet trial; $\mathrm{TG}$, triglyceride; Non-esterified fatty acids

\section{Protocol}

Pretest

The pretest in this study involved using stationary bicycles to measure $\mathrm{VO}_{2 \max }$ and assess exercise intensity. Participants arrived at the laboratory in the afternoon and were asked to wear a heart rate monitor wristband (Polar Electro, Kempele, Finland) and a precalibrated breath-by-breath gas analyzer (Cortex, Metamax 3B, Leipzig, Germany), which were used to collect relevant measurements during the exercise. First, participants' gas samples during the resting state (sitting) were collected for $5 \mathrm{~min}$ to determine their energy expenditure at the resting state. Subsequently, a $\mathrm{VO}_{2 \max }$ test was conducted at a fixed cadence and during an incremental amount of pedal power (in W) on a cycle ergometer. Specifically, cadence was maintained at 70 to 80 rpm under an intensity of $75 \mathrm{~W}$, while the power output was incremented by $25 \mathrm{~W}$ every 3 min until the participant was exhausted. During the test period, the oxygen amount, partial pressure of oxygen $\left(\mathrm{PO}_{2}\right)$, partial pressure of carbon dioxide $\left(\mathrm{PCO}_{2}\right)$, energy expenditure, and heart rate were recorded at each stage to calculate the amount of energy expended at $66 \% \mathrm{VO}_{2 \max }$ and the usage of carbohydrate and fat. The fat and carbohydrate oxidation rates were calculated using the following formula [14]:

$$
\begin{aligned}
& \text { Fat oxidation }(\mathrm{g} / \mathrm{min})=1.695 \times \mathrm{VO}_{2}-1.701 \times \mathrm{VCO}_{2} \\
& \text { Carbohydrate oxidation }(\mathrm{g} / \mathrm{min})=4.585 \times \mathrm{VCO}_{2}-3.226 \times \mathrm{VO}_{2}
\end{aligned}
$$

\section{Formal experiment}

The experiment was conducted over 2 days. Four days before the first formal experiment, a nutritionist individually provided all of the participants with diet-related knowledge and asked them to avoid ingesting an excessive amount of fat and calories as well as alcohol and caffeine. To facilitate dietary control, the participants were asked to record the meals they had ingested during the 3 days preceding the formal experiment and to ingest the same meals 3 days before the subsequent formal experiment. All participants were also asked to avoid excessive physical activities and heavy training 3 days before the formal experiment. Participants arrived at the laboratory between 08:00 and 09:00 in the morning on the first day of the formal experiment. They rested for 10 min before putting on a polar watch and gas analyzer to determine the actual exercise intensity. First, participants rode a cycle ergometer for $12 \mathrm{~min}$ at $66 \% \mathrm{VO}_{2 \max }$, after which the intensity was increased to $85 \% \mathrm{VO}_{2 \max }$ for $4 \mathrm{~min}$ and then decreased to $50 \% \mathrm{VO}_{2 \max }$ for $2 \mathrm{~min}$. Completing these three intensities was considered a cycle, and there was in total five cycles. During the exercise, $200 \mathrm{~mL}$ of drinking water was provided to the participants every $20 \mathrm{~min}$ to prevent dehydration. 
At the end of the exercise, an LF or HF meal was administered to the participants from 09:45-10:45, at 12: 30 , and at 19:00. All meals were prepared by a nutritionist. In the HF trial, the meals had a total calorie intake of $2437.7 \mathrm{kcal}$ and included breakfast (full-cream milk, peanut butter toast, and $8 \mathrm{~g}$ of nuts), lunch (bubble tea, creamy bacon pasta, and kiwi), and dinner (110 g of KFC Chizza and a KFC Zinger). The amounts of fat, protein, and carbohydrate in the three meals were $44 \%$ (119.7 g), $12 \%(71.9 \mathrm{~g})$, and $44 \%(268.2 \mathrm{~g})$ of the total calorie intake, respectively. In the LF trial, the meals had a total calorie intake of $2448.2 \mathrm{kcal}$ and included breakfast ( $40 \mathrm{~g}$ of whey protein, kiwi, banana, Laba congee, and lemon tea), lunch ( $40 \mathrm{~g}$ of whey protein, $200 \mathrm{~g}$ of white rice, $150 \mathrm{~g}$ of sweet mung bean soup, and kiwi), and dinner ( $40 \mathrm{~g}$ of whey protein, boiled vegetables, $200 \mathrm{~g}$ of white rice, a tea egg, black tea, and banana). The amounts of fat, protein, and carbohydrate in the three meals were $6 \%$ (15 g), 20\% (126.3 g), and 74\% (452 g) of the total calorie intake, respectively. The macronutrient consumption for LF and HF were listed in Table 2.

The participants returned to the laboratory at approximately 08:00 AM on the second day of the formal experiment to undertake an OFTT in the fasting state. After 10 min of rest, the participants' fasting blood samples were collected through venipuncture. Subsequently, the participants were given a fixed HF meal and rested in the laboratory for $4 \mathrm{~h}$. Further blood samples were collected at $0.5,1$, 2,3 , and $4 \mathrm{~h}$ after the end of the meal. Postprandial gaseous samples were collected by a precalibrated breath-by-breath gas analyzer (Cortex, Metamax 3B, Leipzig, Germany) from the resting sitting position for $5 \mathrm{~min}$ at each time point to calculate the participants' postprandial fat oxidation rate.

\section{Blood sample collection}

In the experiment, 10-mL blood samples were collected using an intravenous catheter (Venflon 20G cannula,

Table 2 The macronutrient consumption for LF and HF

\begin{tabular}{|c|c|c|c|c|}
\hline & Carbohydrate (g) & Protein (g) & Fat (g) & $\begin{array}{l}\text { Total calorie } \\
\text { (Kcal) }\end{array}$ \\
\hline \multicolumn{5}{|l|}{$\mathrm{HF}$} \\
\hline Breakfast & 41 & 17.6 & 33.5 & 535.9 \\
\hline Lunch & 141.1 & 15.1 & 50.4 & 1078.4 \\
\hline Dinner & 86.1 & 39.2 & 35.8 & 823.6 \\
\hline Total & $268.2(44 \%)$ & $71.9(12 \%)$ & 119.7 (44\%) & 2437.9 \\
\hline \multicolumn{5}{|l|}{ LF } \\
\hline Breakfast & 157 & 31.3 & 3.5 & 784.7 \\
\hline Lunch & 136 & 51 & 6.5 & 806.5 \\
\hline Dinner & 159 & 44 & 5 & 857 \\
\hline Total & 452 (74\%) & $126.3(20 \%)$ & $15(6 \%)$ & 2448.2 \\
\hline
\end{tabular}

LF Low fat diet trial, HF High fat diet trial
Sweden) and three-way connector (Connecta Ltd., Sweden). Samples were collected $30 \mathrm{~min}$ before and immediately and $1,2,3$, and $4 \mathrm{~h}$ after a meal. The blood samples were collected into collection Vacutainers containing ethylenediaminetetraacetic acid (EDTA). To prevent the blood from clotting in the catheter, we used $10 \mathrm{~mL}$ of isotonic saline to clean the catheter. The Vacutainers were centrifuged for $20 \mathrm{~min}$ at $2000 \times \mathrm{g}$ at $4{ }^{\circ} \mathrm{C}$. Blood plasma was extracted and stored at $-80^{\circ} \mathrm{C}$ for subsequent biochemical analysis.

The plasma concentrations of TG, glucose (GLU), glycerol (GLY), and non-esterified fatty acids (NEFA) were determined using an automatic biochemistry analyzer (Hitachi 7020, Tokyo, Japan) and commercially available reagents (GOD-PAP method, Randox, Ireland). The interassay and intra-assay CVs were: TG $(1.9 \%$ \& $0.6 \%$, respectively); GLU (2.2\% \& 3.7\%, respectively); GLY (0.9\% \& $6.4 \%$, respectively); NEFA ( $2.6 \% \& 4.4 \%$, respectively). The plasma concentrations of insulin was determined using an automatic biochemistry analyzer (Elecsys 2010, New York, USA) and commercially available reagents (Electrochemiluminescence immunoassay method, Roche, Switzerland). The inter-assay and intra-assay CVs were 0.83 and $2.6 \%$, respectively.

\section{Oral fat tolerance test (OFTT)}

All the meals provided for the OFTT were designed by a nutritionist and have been used in a previous study $[7,15]$. The meals were composed of toast, butter, cheese, muesli, and fresh cream. The meals provided $1.2 \mathrm{~g}$ of fat per $\mathrm{kg}$ body weight, $1.1 \mathrm{~g}$ of carbohydrate, $0.33 \mathrm{~g}$ of protein, and $16.5 \mathrm{kcal}$ of energy. The nutritional contents of the meals were obtained from the packaging labels. During the experiment, the participants were required to ingest their OFTT meals within $15 \mathrm{~min}$.

\section{Statistical analysis}

All data were presented as mean \pm standard deviation. The t-test was used to test the concentration difference in the area under the curve (AUC) of each dependent variable between the two groups. Two-way ANOVA with repeated measures was performed to analyze the difference in blood biochemical values between the groups and at various time points. A statistically significant difference required posthoc comparison using the Bonferroni method. Significance was defined as $\alpha=0.05$. The $\mathrm{G}^{*}$ Power 3 software program was used to calculate the sufficient sample size with an $\alpha$ value of $5 \%$ and a power of 0.8.The sufficient sample size obtained was eight participants.

\section{Result}

The participants physiological information and fasting plasma biochemistry. 
There were no significantly different between HF and LF in the average heart rate $(p=0.414)$ and energy expenditure $(p=0.527)$ during exercie. The fasting concentrations from the plasma biochemistry did not differ on the morning of Day 2 in all trials (Table 1).
TG concentrations, fat oxidation and carbohydrate oxidation

There were no differences between HF and LF in TG concentrations (trial $\times$ time, $p=0.219$; trial, $p=0.501$; time, $p<0.001$; Fig. 1a), TG AUC ( $p=0.586$; Fig. $1 \mathrm{~b})$,

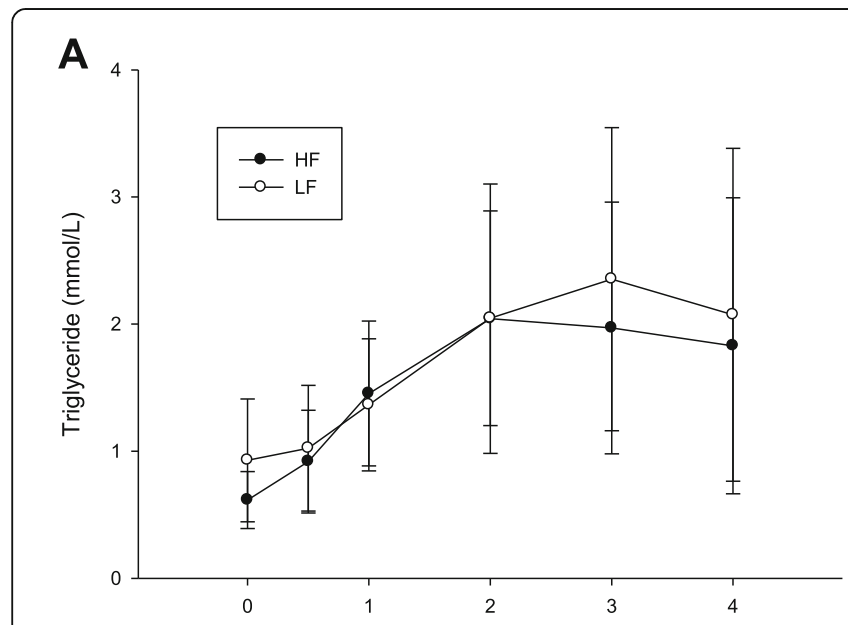

\section{B}

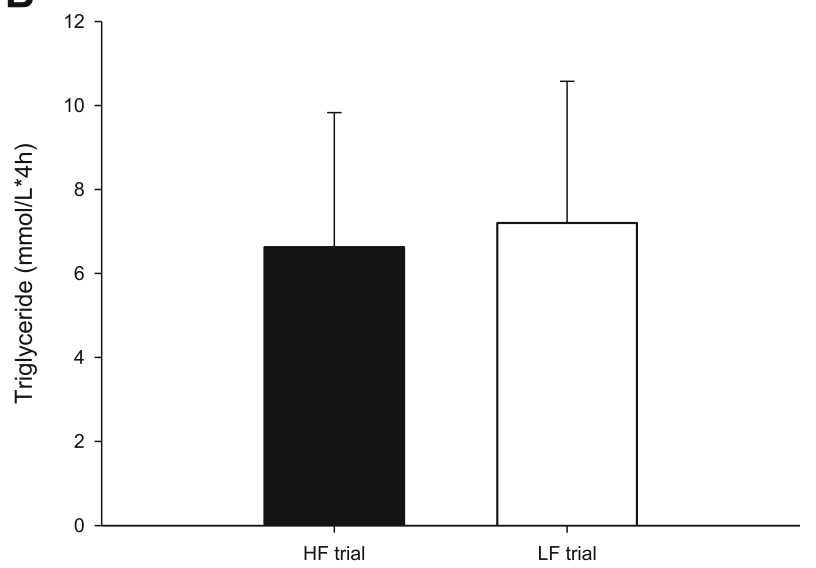

C

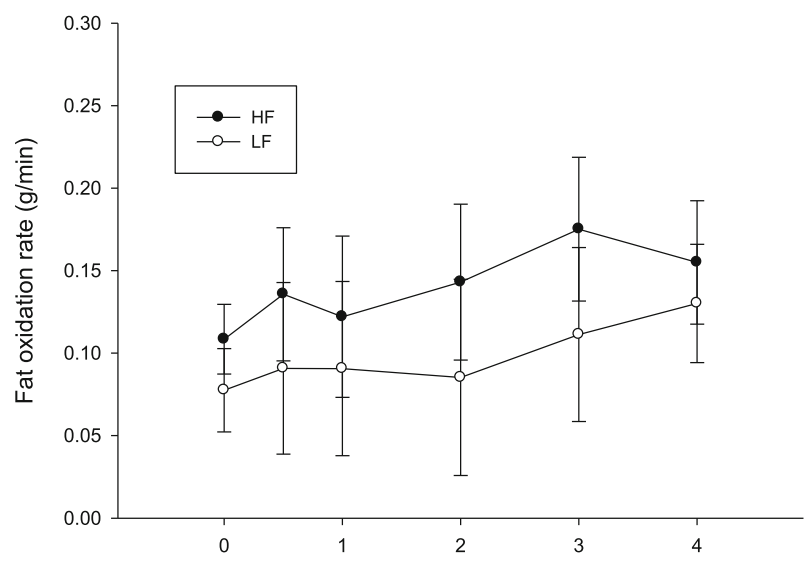

D

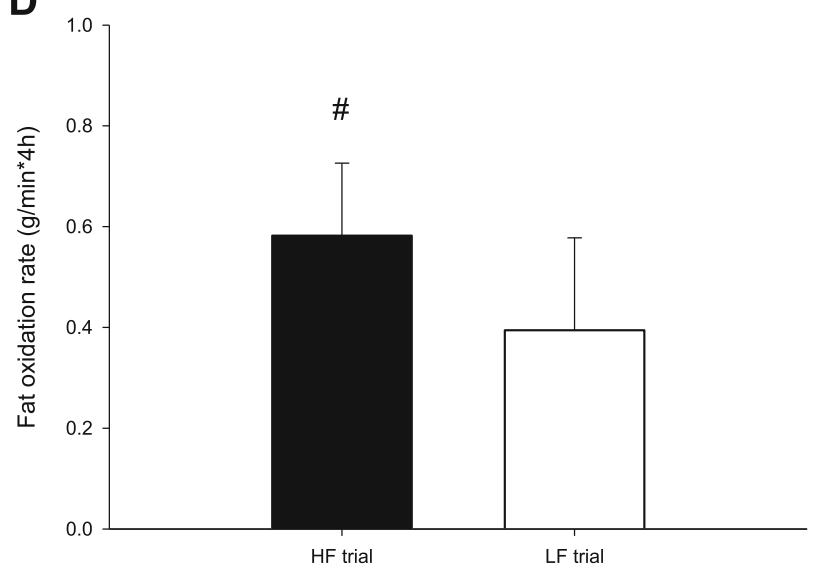

E

$\mathbf{F}$
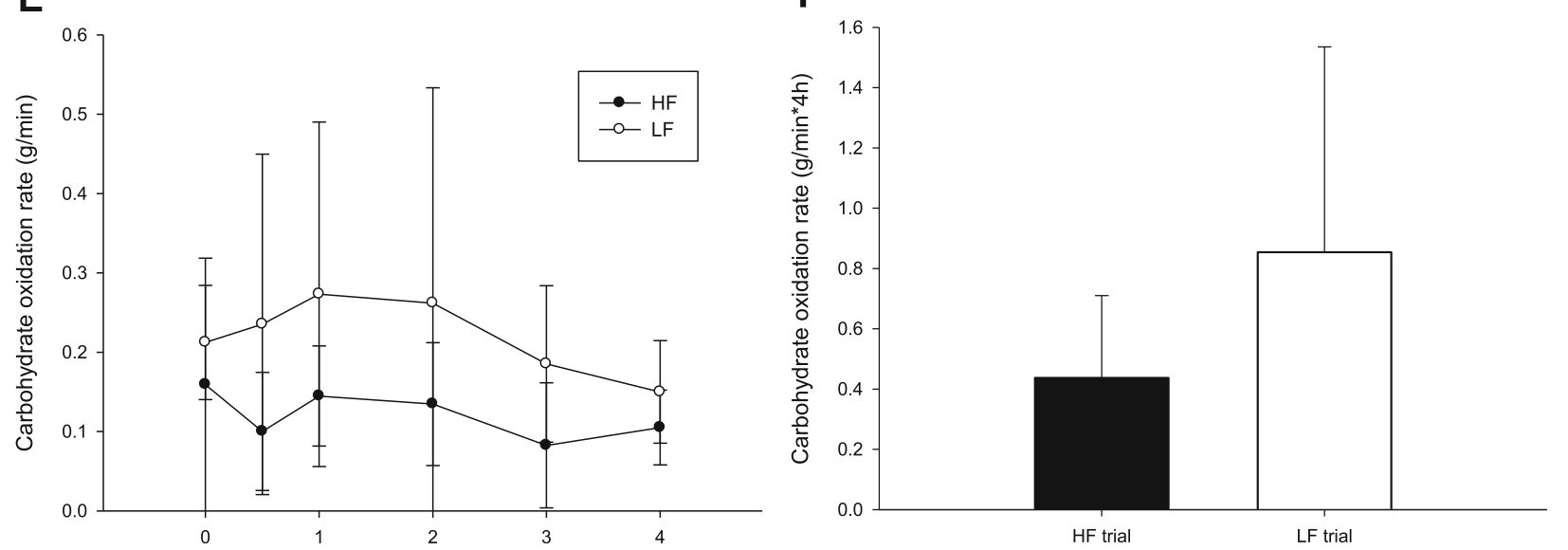

Fig. 1 The postprandial TG concentrations over the $4 \mathrm{~h}(\mathbf{a})$, the TG area under the curve in $4 \mathrm{~h}(\mathbf{b})$, the fat oxidation rate over the $4 \mathrm{~h}(\mathbf{c})$ and the fat oxidation rate area under the curve in $4 \mathrm{~h}(\mathbf{d}) .{ }^{\#} \mathrm{HF}$ was significantly higher than those for the LF 
and fat oxidation rate (trial $\times$ time, $p=0.474$; trial, $p=0.086$; time, $p=0.001$; Fig. $1 \mathrm{c}$ ). Figure $1 \mathrm{~d}$ demonstrates the fat oxidation rate AUC in the HF trial was significantly higher than that in the LF trial $(p=0.045)$. There were no differences between HF and LF in the carbohydrate oxidation rate (trial $x$ time, $p=0.479 ;$ trial, $p=0.387 ;$ time, $p=0.239$; Fig. 1e) and the AUC of carbohydrate oxidation rate $(p=0.216$; Fig. 1f).

\section{GLU and insulin}

Plasma GLU concentrations exhibited no significant differences between trials (trial $\times$ time, $p=0.822$; trial, $p=0.021$; time, $p=0.321$; Fig. $2 \mathrm{a}$ ). Figure $2 \mathrm{~b}$ indicates that the plasma GLU AUC was higher in the HF trial than in the LF trial $(p=0.007)$. There were no differences between HF and LF in the concentrations of insulin (trial $\times$ time, $p=0.503$; trial, $p=0.284$; time, $p<0.001$; Fig. 2 c), but the plasma insulin AUC was higher in the HF trial than in the LF trial ( $p=0.015$; Fig. $2 \mathrm{~d})$.

\section{NEFA and GLY}

Plasma nonesterified fatty acids (NEFA) concentrations has significant interaction (trial $\times$ time, $p=0.042$; Fig. 3a). At $0.5,1$ and $2 \mathrm{~h}$ after the meal in the HF trial were significantly higher than the LF trial $(0.5 \mathrm{~h}, p=0.022 ; 1 \mathrm{~h}, p=$ $0.005 ; 2 \mathrm{~h}, p=0.012$ ). Plasma glycerol (GLY) concentrations has significant interaction (trial $\times$ time, $p=0.038$; Fig. $3 \mathrm{~b})$. At 1, 2, 3 and $4 \mathrm{~h}$ after the meal in the HF trial were significantly higher than the LF trial $(1 \mathrm{~h}, p<0.001$; $2 \mathrm{~h}, p<0.001 ; 3 \mathrm{~h}, p=0.005 ; 4 \mathrm{~h}, p=0.007$ ).

\section{Discussion}

He present study revealed that among exercise interventions with different intensities and the same energy expenditure, HIIE is more capable of reducing the postprandial TG concentrations.

This study revealed that various contents in meals after a 90-min exercise significantly raised the fat oxidation rate after an HF meal the next day, but it did not affect the plasma TG concentration. In addition, the results demonstrated that ingesting an HF meal after
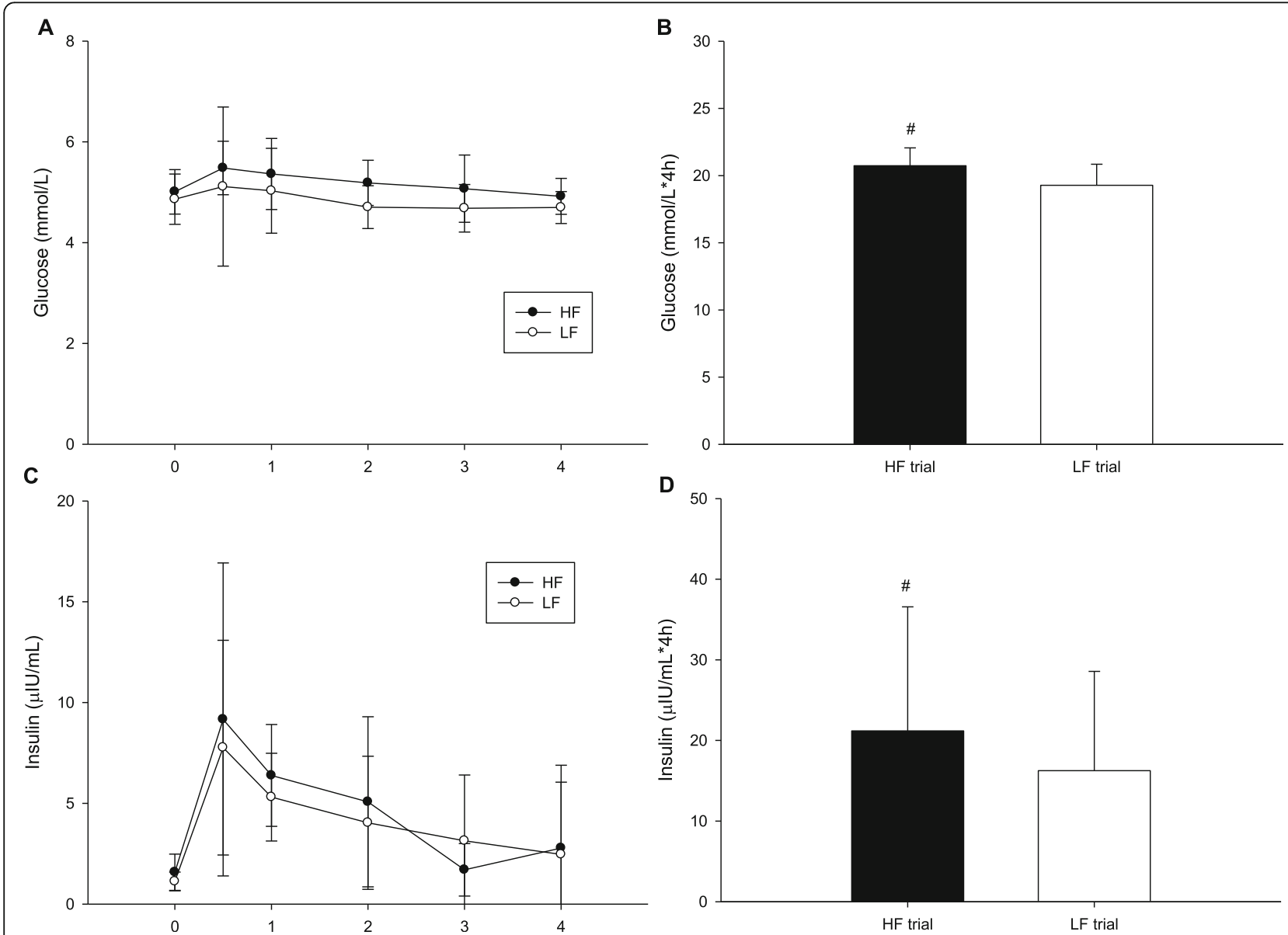

Fig. 2 The postprandial glucose concentrations over the $4 \mathrm{~h} \mathrm{(a),} \mathrm{the} \mathrm{glucose} \mathrm{area} \mathrm{under} \mathrm{the} \mathrm{curve} \mathrm{in} 4 \mathrm{~h}(\mathbf{b})$, the insulin over the $4 \mathrm{~h}(\mathbf{c})$ and the insulin area under the curve in $4 \mathrm{~h}$ (d). " mean HF was significantly higher than those for the LF 

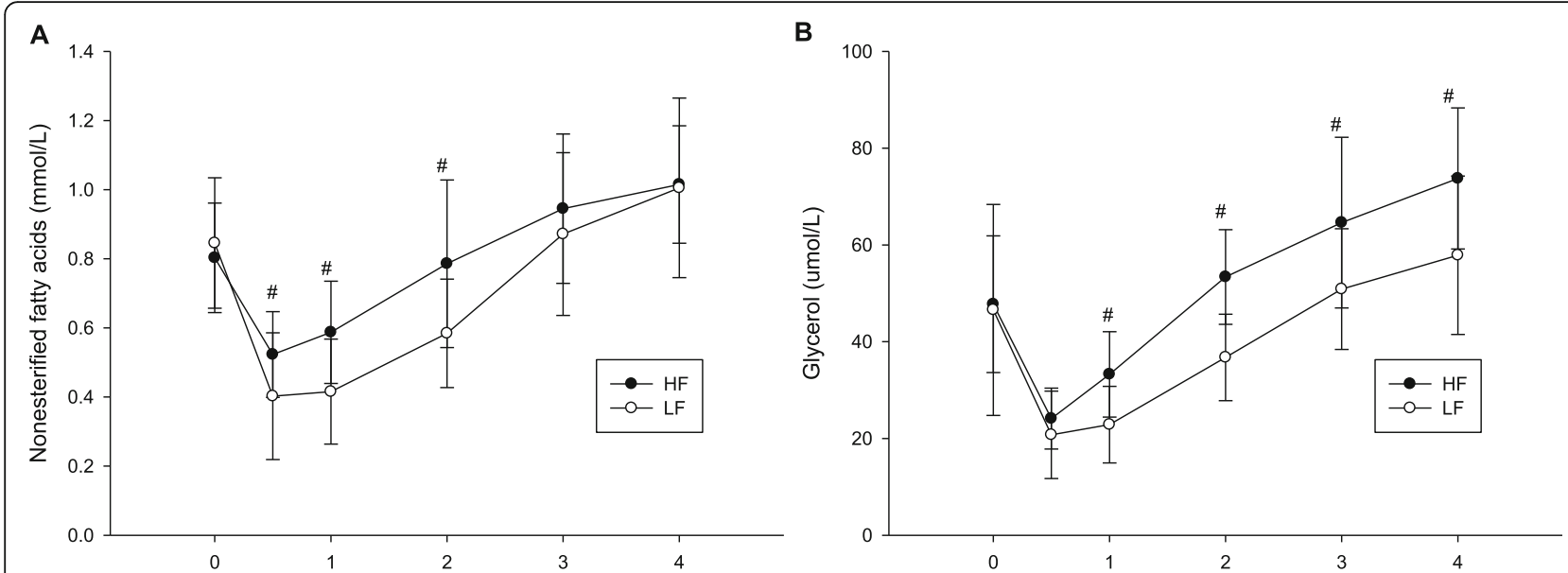

Fig. 3 The postprandial nonesterified fatty acids concentrations over the $4 \mathrm{~h}$ (a) and glycerol concentrations over the $4 \mathrm{~h}$ (b). ${ }^{\#}$ mean $\mathrm{HF}$ was significantly higher than those for the LF

exercise significantly increased postprandial GLU and insulin concentrations.

This study revealed that when the same amount of energy expended during exercise and the same calorie intake on the previous day, meals with dissimilar fat contents did not influence the postprandial TG concentration the next day. In a previous study, low-carbohydrate diets increased the postprandial fat oxidation and decreased the postprandial TG concentration compared with highcarbohydrate diets [10]. However, the fat content in the low-carbohydrate diet trial was $72.2 \%$ in this study. Eating high-fat-content meals in the daily life is difficult. Therefore, we decreased the fat content to $44 \%$ in the meals of the HF trial and successfully increased the postprandial fat oxidation compared with the LF trial, but there were no differences in the postprandial TG concentration between the HF and LF trial. The higher concentration of insulin observed in the HF trial may play a role in the absence of change in the postprandial TG concentration.

The higher insulin concentration in the postprandial period may decrease the LPL activity and influence the postprandial TG response. Previous findings have suggested that ingesting HF meals results in reduced insulin sensitivity [16-18]. Bachmann et al. (2001) fed 12 participants HF and LF meals for 3 days in a row and assessed their insulin sensitivity. The results indicated that the insulin sensitivity fell below $83.3 \pm 5.6 \%$ of the baseline, and insulin sensitivity after an LF diet exhibited a nonsignificant difference [19]. Although we did not calculate the insulin sensitivity in this study, our results demonstrated that the GLU and insulin concentrations of the HF group were considerably higher than those of the LF group, indicating that the HF group was less sensitive to insulin. Based on other data from the present study, the postprandial NEFA and GLY concentrations were higher in the HF trial compared with the LF trial. This may reflect a reduction of the insulin sensitivity in the $\mathrm{HF}$ trial compared with the LF trial. A higher insulin concentration and lower insulin sensitivity have been suggested to decrease the LPL activity and clearance of TG from the blood circulation [20]. Therefore, a higher postprandial insulin response may reduce the positive effect of higher postprandial fat oxidation on postprandial TG concentration.

This study also revealed that the fat oxidation rate significantly increased in the HF trial. In previous studies on the effects of exercise interventions on postprandial lipemia, high-intensity interval training a day before OFTT was found to significantly increase the postprandial fat oxidation rate after an HF meal the next day, and the postprandial TG concentration was also considerably reduced after an OFTT [7]. These findings indicate that an increase in the postprandial fat oxidation rate may influence the postprandial TG concentration. In addition to high-intensity interval training, ingesting HF meals was similarly suggested to elevate the postprandial fat oxidation rate $[10,11]$. However, no studies have investigated whether an increase in fat oxidation rate due to HF meals influences TG concentrations after an HF meal. Although this study revealed an increase in postprandial fat oxidation rate, the postprandial TG concentration was not affected.

The primary limitation of this study is that a control trial (no exercise group) was not used. It is difficult to determine whether the postprandial TG concentration was or not affected in the exercise trial. However, the objective of this study was to investigate the effects of ingesting HF or LF meals on postprandial TG concentration and postprandial fat oxidation after an OFTT the next day. Therefore, a control trial did not appear to be critical for this study. The second limitation of this study was the difference in the protein content among trials. 
The acute effect of the ingestion of additional protein into an HF meal may reduce the postprandial TG concentration [21, 22]. However, no study has investigated the long-term effect of protein ingestion or the effect of protein on the day before the HF meal test. We believe a higher content of protein the day before the HF meal did not influence the results in this study.

\section{Conclusion}

This study revealed that various contents in meals after a 90-min exercise did not influence the postprandial lipemia after an OFTT the next day. Compared with LF meals, HF meals resulted in a higher fat oxidation rate, GLU level, and insulin concentration after an OFTT. Thus, HF diets can cause a reduction in insulin sensitivity. Nevertheless, future studies should consider using the OGTT method to investigate the effects of various meals after exercise on insulin sensitivity.

\section{Acknowledgements}

Thanks for Sports Science Research Center of National Taiwan University of Sport to provide the equipment for this study.

\section{Authors' contributions}

C-HC carried out the experiment, blood analysis and assisted the manuscript preparation. T-JY assisted the data analysis and manuscript preparation. C-HC assisted the experimental design, data analysis and manuscript preparation. $\mathrm{M}-J Z$ carried out the experiment and blood analysis. All authors read and approved the final manuscript.

\section{Funding}

This study as funded by Sports Science Research Center of National Taiwan University of Sport (106DG00107).

\section{Availability of data and materials}

The data analyzed during the present study are available from the corresponding author on reasonable request.

\section{Ethics approval and consent to participate}

This study was approved by the Institutional Review Board of Changhua Christian Hospital (CCH irb No 151221) in Taiwan.. All participants provided written informed consent before participating in the study.

\section{Consent for publication}

Not applicable.

\section{Competing interests}

The authors declare that they have no competing interests.

\section{Author details}

${ }^{1}$ Department of Exercise Health Science, National Taiwan University of Sport, No.16, Sec. 1, Shuang-Shih Rd, Taichung 404, Taiwan. ${ }^{2}$ Department of Physical Education, National Taiwan Normal University, Taipei 106, Taiwan. ${ }^{3}$ Department of Sport Performance, National Taiwan University of Sport, Taichung 404, Taiwan.

Received: 30 May 2019 Accepted: 3 October 2019

Published online: 23 October 2019

\section{References}

1. Nordestgaard BG, Varbo A. Triglycerides and cardiovascular disease. Lancet 2014;384(9943):626-35.

2. Emerson SR, Kurti SP, Snyder BS, Sitaraman K, Haub MD, Rosenkranz SK. Effects of thirty and sixty minutes of moderate-intensity aerobic exercise on postprandial lipemia and inflammation in overweight men: a randomized cross-over study. J Int Soc Sports Nutr. 2016;13(1):26. https://doi.org/10. 1186/s12970-016-0137-8

3. Gabriel B, Ratkevicius A, Gray P, Frenneaux MP, Gray SR. High-intensity exercise attenuates postprandial lipaemia and markers of oxidative stress. Clin Sci. 2012;123(5):313-21.

4. Petitt DS, Cureton KJ. Effects of prior exercise on postprandial lipemia: a quantitative review. Metabolism. 2003;52(4):418-24.

5. Miyashita M, Burns SF, Stensel DJ. Accumulating short bouts of brisk walking reduces postprandial plasma triacylglycerol concentrations and resting blood pressure in healthy young men. Am J Clin Nutr. 2008;88(5): 1225-31.

6. Gill JM, Herd SL, Vora V, Hardman AE. Effects of a brisk walk on lipoprotein lipase activity and plasma triglyceride concentrations in the fasted and postprandial states. Eur J Appl Physiol. 2003;89(2):184-90.

7. Yang T-J, Wu C-L, Chiu C-H. High-intensity intermittent exercise increases fat oxidation rate and reduces postprandial triglyceride concentrations. Nutrients. 2018;10(4):492

8. Gill JM, Hardman AE. Exercise and postprandial lipid metabolism: an update on potential mechanisms and interactions with high-carbohydrate diets. J Nutr Biochem. 2003:14(3):122-32.

9. Sharman MJ, Kraemer WJ, Love DM, Avery NG, Gómez AL, Scheett TP, et al. A ketogenic diet favorably affects serum biomarkers for cardiovascular disease in normal-weight men. J Nutr. 2002;132(7):1879-85

10. Trombold JR, Christmas KM, Machin DR, Van Pelt DW, Chou T-H, Kim I-Y, et al. Postexercise macronutrient intake and subsequent postprandial triglyceride metabolism. Med Sci Sports Exerc. 2014:46(11):2099-106.

11. Schrauwen P, Lichtenbelt WDVM, Saris WH, Westerterp KR. Fat balance in obese subjects: role of glycogen stores. Am J Physiol Endocrinol Metab. 1998;274(6):E1027-233

12. Harrison M, O'Gorman DJ, McCaffrey N, Hamilton MT, Zderic TW, Carson BP, et al. Influence of acute exercise with and without carbohydrate replacement on postprandial lipid metabolism. J Appl Physiol. 2009;106(3): 943-9.

13. Trombold JR, Christmas KM, Machin DR, Kim I-Y, Coyle EF. Acute highintensity endurance exercise is more effective than moderate-intensity exercise for attenuation of postprandial triglyceride elevation. J Appl Physiol. 2013:114(6):792-800

14. Frayn K. Calculation of substrate oxidation rates in vivo from gaseous exchange. J Appl Physiol. 1983;55(2):628-34.

15. Chiu C-H, Burns SF, Yang T-J, Chang Y-H, Chen Y-L, Chang C-K, et al. Energy replacement using glucose does not increase postprandial lipemia after moderate intensity exercise. Lipids Health Dis. 2014;13(1):177.

16. Riccardi G, Giacco R, Rivellese A. Dietary fat, insulin sensitivity and the metabolic syndrome. Clin Nutr. 2004:23(4):447-56.

17. Hancock CR, Han D-H, Chen M, Terada S, Yasuda T, Wright DC, et al. Highfat diets cause insulin resistance despite an increase in muscle mitochondria. Proc Natl Acad Sci. 2008;105(22):7815-20.

18. Seghieri $M$, Trico $D$, Natali $A$. The impact of triglycerides on glucose tolerance: lipotoxicity revisited. Diabetes Metab. 2017;43(4):314-22.

19. Bachmann OP, Dahl DB, Brechtel K, Machann J, Haap M, Maier T, et al. Effects of intravenous and dietary lipid challenge on intramyocellular lipid content and the relation with insulin sensitivity in humans. Diabetes. 2001; 50(11):2579-84

20. Campbell PJ, Carlson MG, Hill JO, Nurjhan N. Regulation of free fatty acid metabolism by insulin in humans: role of lipolysis and reesterification. Am J Phys. 1992;263(6):1063-9.

21. Holmer JJ, Mortensen LS, Astrup A, de Vrese M, Holst JJ, Thomsen C, Hermansen K. Acute differential effects of dietary protein quality on postprandial lipemia in obese non-diabetic subjects. Nutr Res. 2013;33(1): 34-40.

22. Westphal S, Kästner S, Taneva E, Leodolter A, Dierkes J, Luley C. Postprandial lipid and carbohydrate responses after the ingestion of a casein-enriched mixed meal. Am J Clin Nutr. 2004;80(2):284-90.

\section{Publisher's Note}

Springer Nature remains neutral with regard to jurisdictional claims in published maps and institutional affiliations. 